\title{
SLOWLY-VARYING BIFURCATION THEORY IN DISSIPATIVE SYSTEMS
}

\author{
J. P. DENIER AND R. GRIMSHAW'
}

(Received 7 March 1988; revised 19 December 1988)

\begin{abstract}
Systems of coupled nonlinear differential equations with an externally controlled slowly-varying bifurcation parameter are considered. Canonical equations governing the transition between bifurcated solutions are derived by making use of methods of "steady" bifurcation theory. It is found that, depending on the initial amplitudes, the solutions of the transition equations are either asymptotically equivalent to the bifurcated solutions or the solutions develop algebraic singularities at some positive finite time. These singularities correspond to a transition to a solution of a fully nonlinear problem.
\end{abstract}

\section{Introduction}

The behaviour of systems of first order ordinary differential equations of the form

$$
d u / d t=F(\mu, u)
$$

where $u$ is a real $n$-dimensional vector, $F(\mu, u)$ is analytic in both arguments and the parameter $\mu$ is a slowly-varying function of the time $t$ :

$$
\mu=\mu(\varepsilon t), \quad 0<\varepsilon \ll 1
$$

has recently received some attention. The object of such a study is to determine how the bifurcated solutions of (1.1) are approached as the parameter $\mu$ varies through a bifurcation point, $\mu=0$ at $t=0$. Haberman [4] has studied the problem of the transition through a bifurcation point of the slowly-varying bifurcation parameter for algebraic bifurcation problems of both first and second order nonlinear ordinary differential equations. By employing the method of matched asymptotic expansions Haberman shows that

\footnotetext{
${ }^{1}$ School of Mathematics, The University of New South Wales, Kensington, NSW, 2033, Australia.

(C) Copyright Australian Mathematical Society 1990, Serial-fee code 0334-2700/90
} 
for the transcritical bifurcation the equation governing the transition has the form

$$
d v / d s=(v-\alpha s)(v-\beta s)
$$

while for the pitchfork bifurcation the equation governing the transition is of the form

$$
d v / d s=s v-v^{3} .
$$

By considering such equations Haberman demonstrates that the transition to the bifurcated solutions occurs at some finite time after the critical value $(t=0)$ at which "static" bifurcation occurs. In [11] Schecter supplies a proof of Haberman's conjecture that a slowly-varying system which admits a trivial solution, $u=0$, can exist in an unstable state for some finite interval of time.

The physical significance of problems with a time-dependent bifurcation parameter is highlighted in $[2,5,7]$. Motivated by problems in laser physics, Erneux and Mandel [2] consider bifurcation problems of the form

$$
d v / d s=k v^{m}+\mu(\varepsilon t)+\delta
$$

for the cases $m=2,3$ and $k= \pm 1$ and for $\delta$ both zero and nonzero (here $\delta$ is an imperfection parameter). Hall [5] shows that equation (1.3) arises in the study of the nonlinear stability of slowly-varying viscous flows. Kapila [7], in considering the dynamics of a jump in the steady-state response of an Arrhenius system due to the slow passage of the bifurcation parameter through some critical value, shows that the equation governing the transition is of the form (saddle-node bifurcation)

$$
d v / d s=s-v^{2}
$$

In $[8,9]$ Lebovitz and Schaar consider systems in which the parameter $\mu=\mu(\varepsilon t)$ is controlled by the response of the system (1.1) according to

$$
d v / d \tau=F(\mu, v), \quad d \mu / d \tau=\varepsilon G(\mu, v), \quad \tau=\varepsilon t .
$$

Rubenfeld [10] considers the case when the evolution equation under consideration is a nonlinear partial differential equation.

The purpose of this work is to generalize the problem as posed by (1.1) to systems of equations of dimension $n \geq 2$. In Section 2 we consider algebraic bifurcation problems of dimension $n \geq 2$. In Section 3 the Hopf bifurcation 
problem is analyzed, while in Section 4 the saddle-node bifurcation is considered. The analysis will show that the canonical equations governing the transition are of the form (1.2)-(1.4).

\section{Algebraic bifurcation problems}

In this section we consider the equation

$$
d u / d t=A(\mu) u+F(\mu, u)
$$

where $u \in \mathbf{R}^{n}$ and $F(\mu, 0)=\partial F(\mu, 0) / \partial u=0$ for all $\mu \in \mathbf{R}$. $A(\mu)$ is an $n \times n$ matrix whose eigenvalues $\lambda_{1}, \ldots, \lambda_{n}$ are assumed to satisfy the following relations,

$$
\begin{aligned}
\lambda_{z}(0)=0, \quad \lambda_{1}^{\prime}(0) & \neq 0 \\
\operatorname{Re} \lambda_{j}<0, \quad \mu=0, \quad 2 & \leq j \leq n .
\end{aligned}
$$

We also define the eigenvectors $\zeta_{j}$ and the adjoint eigenvectors $\nu_{j}$

$$
A(\mu) \zeta_{j}(\mu)=\lambda_{j}(\mu) \zeta_{j}(\mu), \quad A^{\top}(\mu) \nu_{j}(\mu)=\lambda_{j}(\mu) \nu_{j}(\mu) .
$$

In the case when the parameter $\mu$ is constant there is then a bifurcation from the zero solution, $u=0$ to a new equilibrium solution at $\mu=0$.

The parameter $\mu$ is allowed to vary slowly with time $t$ according to

$$
\begin{gathered}
\mu=\mu(\varepsilon t) \\
\mu(0)=0, \quad \mu(0) \neq 0 .
\end{gathered}
$$

First we shall put the linear part of (2.1) into normal form. We introduce a new coordinate $v$ defined by $u=S v$, where $S=S(\varepsilon t)$ is an $n \times n$ matrix. In terms of our new coordinate $v,(2.1)$ may be written as

$$
d v / d t=S^{-1} A S v-S^{-1} S_{t} v+S^{-1} F(\mu, S v) .
$$

Assuming that the matrix $A(\mu)$ in (2.1) is analytic in $\mu$ together with the assumption that the eigenvalue $\lambda_{1}(\mu)$ is distinct and simple ensures that $\lambda_{1}(\mu)$ will be analytic in $\mu$. This ensures that the eigenvector $\zeta_{1}(\mu)$ will be analytic in $\mu$, as will the corresponding adjoint eigenvector $\nu_{1}(\mu)$. The remaining eigenvectors, $\zeta_{k}(k=2,3, \ldots, n)$, ar then automatically orthogonal to the adjoint eigenvector and can be chosen so as to be analytic in $\mu$. Assuming further that the $\zeta_{k}(k=2, \ldots, n)$ are linearly independent we can then choose our transformation matrix $S$ to be $S=\left(\zeta_{1}, \zeta_{2}, \ldots, \zeta_{n}\right)$. Both $S$ and $X^{-1}$ are then assured of being analytic in $\mu$ and hence smooth functions of $t$. With this choice of $S$ we then have

$$
S^{-1} A S=B=\left(\begin{array}{cc}
\lambda_{1} & 0 \\
0 & B_{1}
\end{array}\right)
$$


where $B_{1}$ is an $(n-1) \times(n-1)$ matrix, analytic in $\mu$, whose eigenvalues have (locally) negative real parts. Our transformed system now has the form

$$
\frac{d v}{d t}+B v-\varepsilon X^{-1} S_{\tau}+S^{-1} F(\mu, S v), \quad \tau=\varepsilon t .
$$

To reduce (2.2) to a form which can be analyzed we make use of the method of projection (e.g. see [6]). To this end we write

$$
v=a \zeta_{1}+z
$$

where

$$
B \zeta_{1}=\lambda_{1} \zeta_{1}, \quad\left\langle z, \eta_{1}\right\rangle=0,
$$

and $\langle x, y\rangle=x_{i} \bar{y}_{i}$ is the usual Euclidean dot product. Here $a=\left\langle v, \eta_{1}\right\rangle$ is the projection of $v$ onto the vector space spanned by the eigenvector $\zeta_{1} ; \eta_{1}$ is the adjoint eigenvector of $B$ corresponding to the eigenvector $\lambda_{1}$ i.e.

$$
\eta_{1}=\zeta_{1}=(1,0, \ldots, 0)^{T}, \quad\left\langle\zeta_{1}, \eta_{1}\right\rangle=1 .
$$

Substituting (2.3) into equation (2.2) yields, after some rearrangement

$$
\begin{gathered}
d a / d t=\lambda_{1} a-\varepsilon a \varphi_{1}-\varepsilon b z+F_{1}(\mu, a)+N_{1}(\mu, a, z), \\
d z / d t=\left(B_{1}+\varepsilon B_{2}\right) z+\varepsilon a \varphi_{2}+F_{2}(\mu, a)+N_{2}(\mu, a, z)
\end{gathered}
$$

where

$$
\begin{gathered}
\varphi_{1}=\left\langle S^{-1} S_{\tau} \zeta_{1}, \eta_{1}\right\rangle, \\
\varphi_{2}=\left\langle S^{-1} S_{\tau} \zeta_{1}, \eta_{1}, \zeta \zeta_{1}-S^{-1} S-\tau \zeta_{1}\right. \\
b z=\left\langle S^{-1} S_{\tau} z, \eta_{1}\right\rangle, \\
B_{2} z=\left\langle S^{-1} S_{\tau} z, \eta_{1}\right\rangle \zeta_{1}-S^{-1} S_{\tau} z, \\
V_{1}(\mu, a)+N_{1}(\mu, a, z)=\left\langle S^{-1} F(\mu, S v), \eta_{1}\right\rangle, \\
F_{2}(\mu, a)+N_{2}(\mu, a, z)=S^{-1} F(\mu, S v)-\left\langle S^{-1} F(\mu, S v), \eta_{1}\right\rangle \zeta_{1},
\end{gathered}
$$

and $F_{i}(\mu, a)=O\left(a^{2}\right), N_{i}(j=1,2)$ are nontrivial in $z$. Next we note that the terms explicit in $a$ alone in (2.4b) can be removed to higher order, without affecting the leading order terms in $(2.4 \mathrm{a})$, by making a succession of coordinate changes of the form $z=z+f(a, \tau)$. Thus the terms explicit in $a$ in $(2.4 \mathrm{~b})$ can be transformed to $\varepsilon^{n}$ and $a^{p}$ for arbitrarily large positive integers $n$ and $p$.

Since, by assumption, the eigenvalues of $B_{1}+\varepsilon B_{2}$ are negative (for $\varepsilon$ sufficiently small) one easily obtains estimates on the magnitude of $z$ as

$$
z=O\left(\varepsilon^{n}|a|,|a|^{p}\right) .
$$

Thus, (2.4a) may be rewritten as

$$
d a / d t=\lambda_{1} a+\sigma_{1} a^{2}+O\left(\varepsilon a, a^{3}\right)
$$




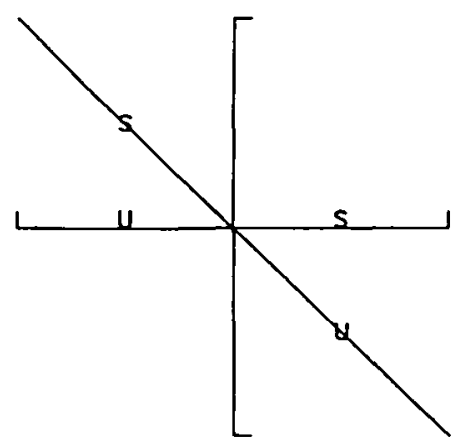

(a)

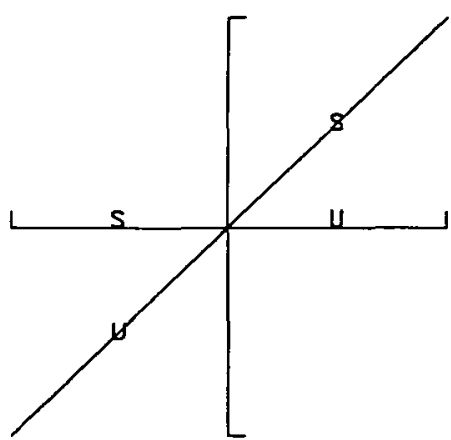

(b)

FIGURE 1. Bifurcation diagrams for transcritical bifurcation. Here $S$ denotes stable and $U$ unstable.

where $\sigma=\sigma(\varepsilon t)$; for simplicity we assume that $\sigma_{1}(0)$ is nonzero (this in fact corresponds to the transcritical bifurcation, whereas the pitchfork bifurcation occurs when the first nonlinear term in (2.5) is $\left.O\left(a^{3}\right)\right)$.

Equation (2.5) has the outer asymptotic solutions $a=0, a \sim-\lambda_{1} / \sigma_{1}$. We wish to examine the possible transition behaviour between these outer asymptotic solutions as $\lambda_{1}$ changes sign. There are, in fact, two distinct cases to consider, depending on the sign of $\lambda_{1} / \sigma_{1}$. These two cases are represented in Figure 1 (here "S" denotes stable and " $U$ " denotes unstable, in analogy with the steady bifurcation problem).

Case $\mathrm{i}$ ), as represented by Figure $1(\mathrm{a})$, is characterised by $\lambda_{1}=-\lambda \tau, \sigma_{1}=$ $-\sigma$ (to leading order). We will denote this case by straight to zero transition. Case ii), corresponding to Figure $1 \mathrm{~b}$, is characterised by $\lambda=\lambda \tau, \sigma_{1}=-\sigma$. We will denote this case by zero to straight transition. In both cases $\lambda, \sigma>0$ and we are assuming that $\mu=\tau$, (the canonical case). We will restrict our attention to Case i) and mention only briefly the behaviour in Case ii).

Straight to zero transition. In Case i), we are interested in the transition from the stable outer solution $a \sim-\lambda_{1} / \sigma_{1}(\tau \rightarrow-\infty)$. To obtain the equation governing the transition we rescale

$$
a=\left(\varepsilon \lambda / \sigma^{2}\right)^{1 / 2} X+O(\varepsilon), \quad \tau=(\varepsilon / \lambda)^{1 / 2} T, \quad X=O(1) .
$$

To leading order in $\varepsilon$ we obtain from (2.5)

$$
d X / d T=-T X-X^{2} \text {. }
$$

Note that $X=0$ is a solution of $(2.6 \mathrm{~b})$ for all $T$.

We note that $(2.6 \mathrm{~b})$ is nothing more than a logistic equation with time dependent coefficients. The transformation $X=\varphi^{-1}$ converts (2.6b) into an 
equation linear in $\varphi$ which upon solving for $\varphi$ yields

$$
X=\exp \left(-T^{2} / 2\right)\left[C+\int_{-\infty}^{T} \exp \left(-v^{2} / 2\right) d v\right]^{-1},
$$

where $C$ is a constant of integration.

In the case $C=0$ we have

$$
X=\exp \left(-T^{2} / 2\right)\left[\int_{-\infty}^{T} \exp \left(-v^{2} / 2\right) d v\right]^{-1},
$$

which has the asymptotic behaviour

$$
X \sim \begin{cases}-T & \text { as } T \rightarrow-\infty \\ 0 & \text { as } T \rightarrow+\infty\end{cases}
$$

Thus, in the case $C=0,(2.8)$ describes the transition from the stable branch of the outer solution $X \sim-T(T \rightarrow-\infty)$ to the stable branch of the outer solution $X=0$ as $T \rightarrow+\infty$.

In general, however, $C \neq 0$. In this case the solutions (2.7) of (2.6) can be classified as follows depending on the value of $C$.

(a) $0<-C<\int_{-\infty}^{\infty} e^{-v^{2} / 2} d v=\sqrt{\pi}$. In this case there exists a $T_{1}$ such that

$$
-C=\int_{-\infty}^{T_{1}} e^{-v^{2} / 2} d v
$$

There are then two possible cases for the solutions (2.7) of (2.6). Either

$$
X \rightarrow \begin{cases}+\infty & \text { as } T \rightarrow T_{1}^{+} \\ 0 & \text { as } T \rightarrow+\infty\end{cases}
$$

(corresponding to a transition from a fully nonlinear outer solution to the stable branch of the zero solution) or

$$
X \rightarrow \begin{cases}0 & \text { as } T \rightarrow-\infty \\ -\infty & \text { as } T \rightarrow T_{1}^{-}\end{cases}
$$

(corresponding to a transition from the unstable branch of the zero solution to a fully nonlinear outer solution).

(b) $-C>\int_{-\infty}^{\infty} e^{-v^{2} / 2} d v$. In this case $X<0$ for all $T$ and $X \rightarrow 0$ as $T \rightarrow \pm \infty$. This corresponds to a transition from the unstable branch of the zero solution to the stable branch of the zero solution.

(c) $C>0$. In this case $X>0$ for all $T$ and $X \rightarrow 0$ as $T \rightarrow \pm \infty$ again corresponding to a transition from the unstable branch of the zero solution to the stable branch of the zero solution.

In Figure 2 we present numerical solutions of the differential equation (2.6b) exhibiting the general behaviour of the solutions (2.7) for the case $C \neq 0$. 


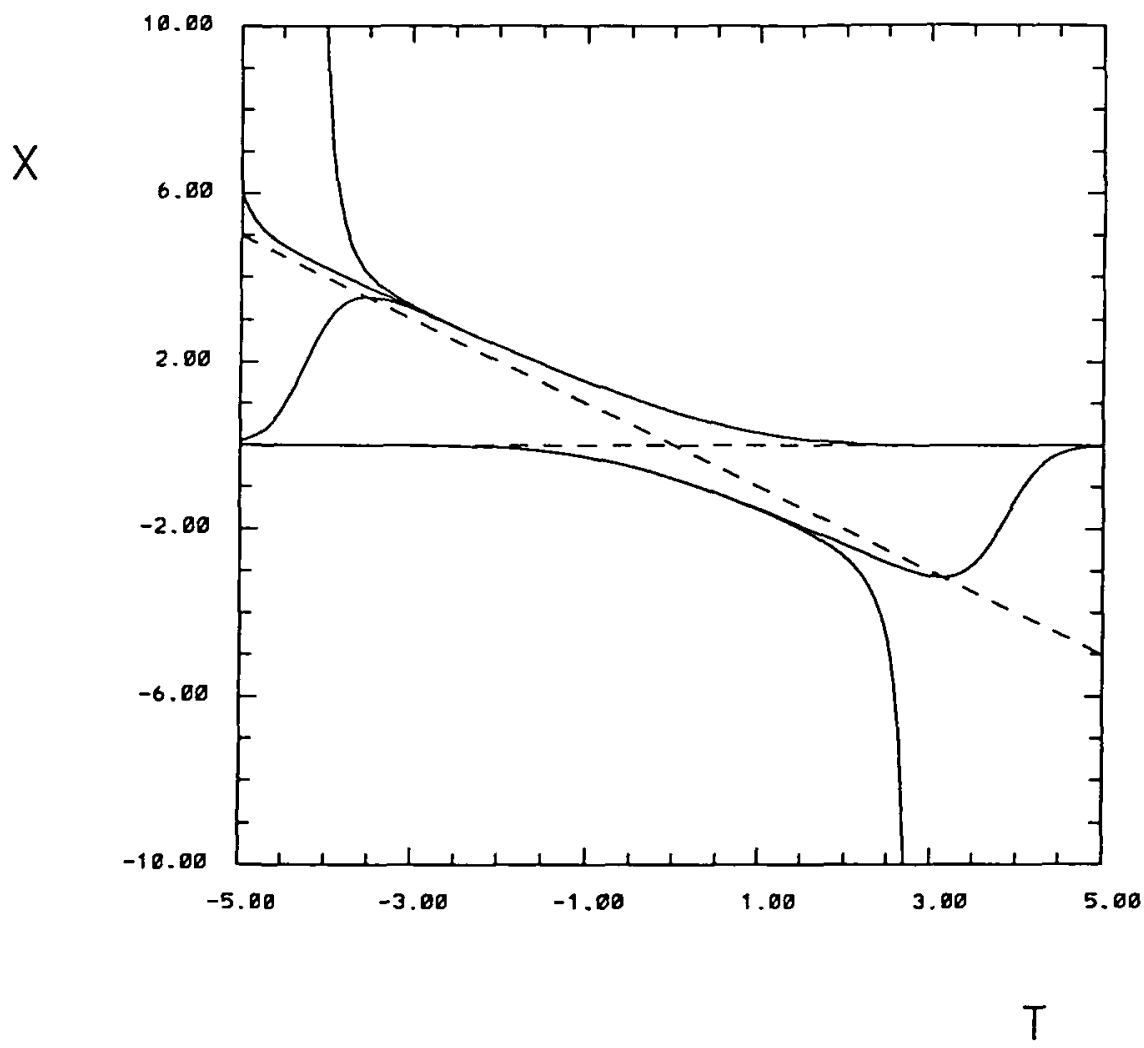

Figure 2. Numerical solutions of (2.6b) (solid lines). Dashed lines $X=0$ and $X=-T$.

Zero to straight transition. In Case ii), as represented by Figure 1(b), we are interested in a transition from the stable zero solution (for $\tau<0$ ) to the stable branch of the bifurcated solution. This case has been studied by Haberman [4] who shows that for $\tau<0$ the evolution is effectively governed by a linear equation. This linear equation is valid until some time $\tau=\tau_{2}>0$ at which there is a rapid transition to the stable branch of the bifurcated solution. As a consequence the solution to (2.5) is able to exist in an unstable state for some finite time $0<\tau<\tau_{2}$. 


\section{Slowly-varying Hopf bifurcation problems}

\subsection{Two dimensional Hopf bifurcation}

In this section we consider equations of the same form as (2.1) except that now $u \in \mathbf{R}^{2}$ and $A(\mu)$ is a $2 \times 2$ matrix with complex conjugate eigenvalues satisfying

$$
\begin{gathered}
\lambda_{1}(\mu)=\alpha_{1}(\mu)+i \beta_{1}(\mu)=\overline{\lambda_{2}(\mu)} \\
\alpha_{1}(0)=0, \quad \alpha_{1}^{\prime}(0) \neq 0, \quad \beta_{1}(0)=\beta_{0} \neq 0
\end{gathered}
$$

(bar denotes the complex conjugate). Following Section 2 we allow $\mu$ to be a slowly-varying function of the time $t$. Introducing a new variable $v$ defined as in Section 2 we choose our transformation matrix $S$ such that $S^{-1} A S=B$ where

$$
B=\left(\begin{array}{cc}
\alpha & \beta \\
-\beta & \alpha
\end{array}\right)
$$

As in Section 2, $S$ and $S^{-1}$ can be chosen to be smooth functions of $\tau=\varepsilon t$ (provided that $A(\mu)$ is analytic in $\mu$ ). The transformed system takes the form

$$
d v / d t=B v-\varepsilon S^{-1} S_{\tau} v+S^{-1} F(\mu, S v) .
$$

Again we project $v$ onto the eigenspace spanned by the eigenvector $v_{1}$

$$
B \zeta_{1}=\lambda_{1} \zeta_{1}
$$

by writing

$$
v=a \zeta_{1}+\bar{a} \bar{\zeta}_{1}
$$

where $\zeta_{1}=(1, i)^{\top}$, (the adjoint eigenvector is $\eta_{1}=\bar{\zeta}_{1} / 2$ so that $\left\langle\zeta_{1}, \eta_{1}\right\rangle=1$ ). Substituting (3.1) into the transformed equation, using the fact that $\zeta_{1}$ is time independent, and taking the inner product with $\eta_{1}$ yields

$$
d a / d t=\lambda_{1} a-\varepsilon\left(a \varphi_{3}+\bar{a} \varphi_{4}\right)+G(\mu, a, \bar{a})
$$

where

$$
\begin{gathered}
\varphi_{3}=\left\langle S^{-1} S_{\tau} \zeta_{1}, \eta_{1}\right\rangle \\
\varphi_{4}=\left\langle X^{-1} S_{\tau} \bar{\zeta}_{1}, \eta_{1}\right\rangle \\
G(\mu, a, \bar{a})=\left\langle S^{-1} F(\mu, S v), \eta_{1}\right\rangle
\end{gathered}
$$

and $G(\mu, a, \bar{a})$ is homogeneous in both $a$ and $\bar{a}$.

To get (3.2) into a form which is amenable to analysis we reduce the function $G(\mu, a, \bar{a})$ to its normal form (for a concise review of normal form theory see [3]). This is achieved by introducing a new variable

$$
a^{\prime}=a+f(a, \bar{a}, \tau)
$$


where the function $f(a, \bar{a}, \tau)$ is homogeneous of degree 2 in $a$. By suitably choosing the nonlinear function $f$ one can remove all quadratic (and in fact all quartic) terms from $G$ and all the cubic terms except for the term $a^{\prime}\left|a^{\prime}\right|^{2}$. The resulting normal form for $G$ is then $G=\sigma_{1} a^{\prime}\left|a^{\prime}\right|^{2}+O\left(\left|a^{\prime}\right|^{5}\right)$. Thus, (3.2) may be reduced to the following canonical form:

$$
d a^{\prime} / d t=\lambda_{1} a^{\prime}+\sigma_{1} a^{\prime}\left|a^{\prime}\right|^{2}+O\left(\varepsilon\left|a^{\prime}\right|,\left|a^{\prime}\right|^{5}\right)
$$

where $\sigma_{1}=\sigma_{1}(\varepsilon t)$ is (generically) nonzero at $\tau=0$. By defining $a^{\prime}=\operatorname{Re}^{i \theta}$, where both the amplitude $R$ and the phase $\theta$ are assumed to be real, we obtain upon equating real and imaginary components in (3.3)

$$
\begin{gathered}
d R / d t=\alpha_{1} R+\sigma_{2} R^{3}+O\left(\varepsilon R, R^{5}\right) \\
d \theta / d t=\beta_{1}+\sigma_{3} R^{2}+O\left(\varepsilon R, R^{4}\right)
\end{gathered}
$$

where we have used $\lambda_{1}=\alpha_{1}+i \beta_{1}$ and $\sigma_{1}=\sigma_{2}+i \sigma_{3}$.

We restrict our attention to the amplitude equation (3.4a). This equation possesses leading order outer asymptotic solutions

$$
R=0 \text { or } R^{2} \sim-\alpha_{1} / \sigma_{2} .
$$

We wish to examine the possible transition behaviour between these outer asymptotic solutions as $\alpha_{1}$ changes sign. There are two distinct cases to consider depending on the sign of $\alpha_{1} / \sigma$. The two cases are represented in Figure 3 (here " $S$ " denotes stable and " $U$ " denotes unstable, in analogy with the steady bifurcation problem).

Case i), as represented by Figure 3(a), is characterised by $\alpha_{1}=-\alpha \tau, \sigma_{2}=-\sigma$ (to leading order). We will call this case the parabolic to zero transition. Case ii), corresponding to Figure $3(\mathrm{~b})$, is characterised by $\alpha_{1}=\alpha \tau, \sigma_{2}=-\sigma$. We will call this case the zero to parabolic transition. In both cases $\alpha, \sigma>0$ and

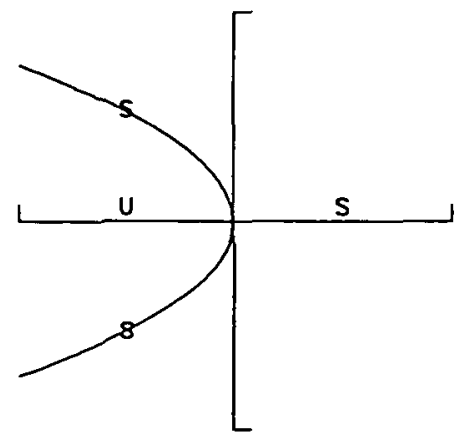

(a)

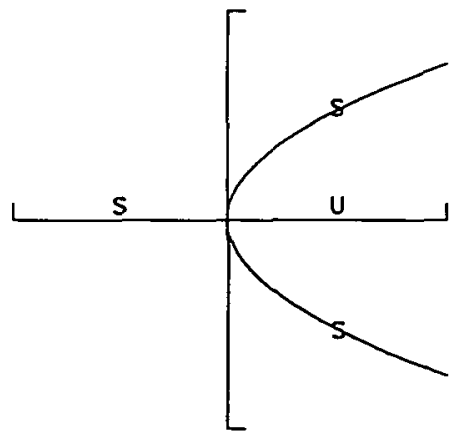

(b)

FIGURE 3. Bifurcation diagrams for Hopf bifurcation, (a) subcritical (b) supercritical. Here $S$ denotes stable and $U$ denotes unstable. 
we assume that $\mu=\tau$ (the canonical case). We will restrict our attention to Case i) and mention only briefly Case ii).

Parabolic to zero transition. In this case we are interested in the behavior of solutions which initially lie in a neighbourhood of the outer solution $R^{2}=-\tau$ (for $\tau<0$ ). To this end we rescale $R$ and $\tau$ :

$$
\bar{K}=\left(\varepsilon \alpha / \sigma^{2}\right)^{1 / 4} \bar{X}, \quad \tau=(\varepsilon / \alpha)^{1 / 2} \bar{T}, \quad \bar{X}, T=\hat{O}(\mathrm{i}) .
$$

Then from (3.4a) we obtain

$$
d X / d T=-T X-X^{3} .
$$

Equation (3.6b) arises in the study of the nonlinear stability of slowly-varying flows, (see Hall [5]).

Defining $Y=X^{2}$ we obtain from (3.6b)

$$
d Y / d T=-2 T Y-2 Y^{2} .
$$

which is equivalent, up to rescaling, to (2.6b). Again, (3.6c) is an example of an exactly solvable Bernoulli equation whose solution is given by

$$
X^{2}=Y=\exp \left(-T^{2}\right)\left[C+2 \int_{T_{0}}^{T} \exp \left(-v^{2}\right) d v\right]^{-1}
$$

In the case $C=0$ we have

$$
X^{2}=\exp \left(-T^{2}\right)\left[2 \int_{T_{0}}^{T} \exp \left(-v^{2}\right) d v\right]^{-1}
$$

which is the asymptotic behaviour

$$
X \sim \begin{cases}|T|^{1 / 2} & \text { as } T \rightarrow-\infty \\ 0 & \text { as } T \rightarrow+\infty\end{cases}
$$

and thus corresponds to a transition from the stable branch of the outer solution $X \sim|T|^{1 / 2}$ to the stable branch of the zero solution as $T \rightarrow \infty$. In the general case $C \neq 0$ the solutions (3.7) of (3.6c) can be classified as in Section 2.

In Figure 4 we present numerical solutions of (3.6b) exhibiting the general behaviour of solutions (3.7) when $C \neq 0$.

Zero to parabolic transition. In this case we are interested in the transition from the stable zero solution $T<0$ to the stable branch of the bifurcated solution. This case has been analyzed by Haberman [4] who shows that the evolution of a solution of (3.4a) is governed by a linear equation until some positive time $\tau^{*}$ at which there is a rapid transition to the stable branch of the bifurcated solution. Haberman shows that there is a delay in the approach to 

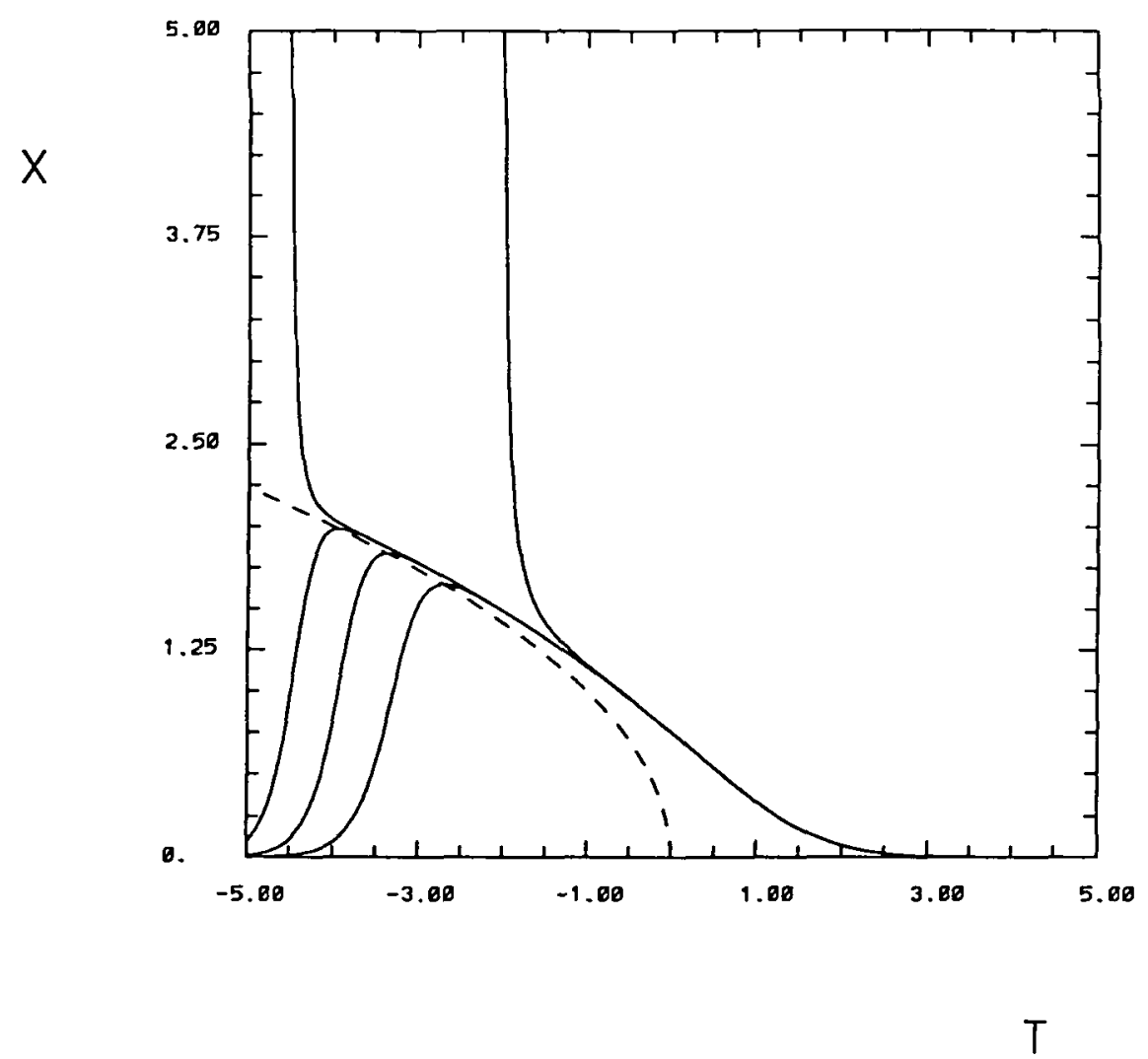

FIGURE 4. Numerical solutions of (3.6b) (solid lines). Dashed line $X=|T|^{1 / 2}$.

the stable outer solution. As a consequence, the solution exists in an unstable state for some finite time $\tau, 0<\tau<\tau^{*}$.

We now consider the general problem

$$
d u / d t=A(\mu) u+F(\mu, u) \quad u \in \mathbf{R}^{n}(n \geq 3)
$$

where

$$
F(\mu, 0)=\partial F(\mu, 0) / \partial u=0, \quad \text { for all } \mu \in \mathbf{R} .
$$

We assume that $A(\mu)$ has eigenvalues $\lambda_{i}(\mu),(i=1,2, \ldots, n)$ satisfying

$$
\begin{gathered}
\lambda_{1}=\alpha_{1}+i \beta_{1}=\bar{\lambda}_{2} \\
\alpha_{1}(0)=0, \quad \begin{array}{c}
\alpha_{1 \mu}(0)=\alpha \neq 0 \quad \beta_{1}(0)=\beta_{0} \neq 0 \\
\operatorname{Re} \lambda_{j}(0)<0 \quad j=3, \ldots, n .
\end{array}
\end{gathered}
$$

This characterizes Hopf bifurcation in $\mathbf{R}^{n}$ (see [3], [6]). As in previous sections we allow $\mu$ to vary slowly with respect to the time $t$ (see Section 1). 
Following the procedure of Section 2 we make a smooth (in $t$ ) change of variables $u=S v$ and choose $S$ such that $S=\left(\zeta_{R}, \zeta_{I}, \zeta_{3}, \ldots, \zeta_{n}\right), A(\mu) \zeta_{i}=\lambda_{i}(\mu) \zeta_{i}$ where $\zeta_{R}$ and $\zeta_{I}$ are the real and imaginary components of the eigenvector $\zeta_{1}$. Assuming that $A(\mu)$ is analytic in $\mu$ ensures that $\lambda_{1},(\mu), \zeta_{1}(\mu)$ and $\nu_{1}(\mu)$ (the adjoint eigenvector) are analytic in $\mu$. The $\zeta_{j}(j=3, \ldots, n)$ are then orthogonal to $\nu_{1}(\mu)$ and can clearly be chosen analytic in $\mu$. With this choice of $S$ (3.8) becomes

$$
d v / d t=B v-\varepsilon S^{-1} S_{\tau} v+S^{-1} F(\mu, S v)
$$

where now

$$
B=\left(\begin{array}{cc}
B_{1} & 0 \\
0 & b_{2}
\end{array}\right), \quad B_{1}=\left(\begin{array}{cc}
\alpha & \beta \\
-\beta & \alpha
\end{array}\right)
$$

and $B_{2}$ is an $(n-2) \times(n-2)$ matrix whose eigenvalues $\lambda_{j}(\mu)$ have (locally) negative real components. Defining

$$
\zeta_{1}=(1, i, 0, \ldots, 0)^{\top}, \quad \eta_{1}=1 / 2(1,-i, 0, \ldots, 0)^{\top} \quad\left\langle\zeta_{1}, \eta_{1}\right\rangle=1
$$

(the eigenvector and adjoint eigenvector of $B$ corresponding to eigenvalue $\left.\lambda_{1}(\mu)\right)$ we write $v=a \zeta_{1}+\bar{a} \bar{\zeta}_{1}+z$ with $z$ orthogonal to $\eta_{1}$ and $\bar{\eta}_{1}$. Substituting into (3.9) and taking the inner product of the resulting equation with $\eta_{1}$ and $\bar{\eta}_{1}$ we obtain after some rearrangement

$$
\begin{aligned}
& d a / d t=\lambda_{1} a-\varepsilon\left(a \varphi_{1}+\bar{a} \varphi_{2}\right)-\varepsilon C_{1} z+G_{1}(\mu, a, \bar{a}, z), \\
& d z / d t=B_{2} z+\varepsilon\left(a \varphi_{3}+\overline{a \varphi}_{3}\right)+\varepsilon C_{2} z+G_{2}(\mu, a, \bar{a}, z)
\end{aligned}
$$

where

$$
\begin{gathered}
\varphi_{1}=\left\langle S^{-1} S_{\tau} \zeta_{1}, \eta_{1}\right\rangle \\
\varphi_{2}=\left\langle S^{-1} S_{\tau} \bar{\zeta}_{1}, \eta_{1}\right\rangle \\
\varphi_{3}=\left\langle S^{-1} S_{\tau} \zeta_{1}, \eta_{1}\right\rangle \zeta_{1}-\left\langle S^{-1} S_{\tau}, \zeta_{1}, \bar{\eta}_{1}\right\rangle \bar{\zeta}_{1}-S^{-1} S_{\tau} \zeta_{1} \\
C_{1} z=\left\langle S^{-1} S_{\tau} z, \eta_{1}\right\rangle \\
C_{2} z=\left\langle S^{-1} S_{\tau} z, \eta_{1}\right\rangle \zeta_{1}+\left\langle S^{-1} S_{\tau} z, \bar{\eta}_{1}\right\rangle \bar{\zeta}_{1}-S^{-1} S_{\tau} z \\
G_{1}=\left\langle S^{-1} F(\mu, S v) \zeta_{1}\right\rangle \\
G_{2}=S^{-1} F(\mu, S v)-\left\langle S^{-1} F(\mu, S v), \bar{\eta}_{1}\right\rangle \zeta_{1}-\left\langle S^{-1} F(\mu, S v), \bar{\eta}_{1}\right\rangle \bar{\zeta}_{1} .
\end{gathered}
$$

As in Section 2 we can remove all terms explicit in $a$ in (3.10b) by making a succession of coordinate transformations $z^{\prime}=z+f(a, \bar{a}, \tau)$. This can be done without affecting the leading order terms in $a$ in (3.10a). Thus the terms explicit in $a$ can be transformed to $\varepsilon^{n} a, a^{p}$ for arbitrarily large integers $n$ and $p$. Making use of the fact that the eigenvalues of $B_{2}+\varepsilon C_{2}$ have negative real parts (for $\varepsilon$ sufficiently small) we can again obtain estimates on the magnitude of $z$ as $z=O\left(\varepsilon^{n}|a|,|a|^{p}\right)$. Thus (3.10a) can be rewritten as

$$
d a / d t=\lambda_{1} a+G(\mu, a, \bar{a})+O(\varepsilon|a|)
$$


where $G(\mu, a, \bar{a})$ is homogeneous of degree $k \geq 2$ in $a$. The function $G(\mu, a, \bar{a})$ can be reduced to its normal form and the analysis of the higher dimensional Hopf bifurcation now parallels that of the two dimensional case.

\section{Saddle-node bifurcation}

In the previous two sections we have considered bifurcation problems in which the bifurcation is from the trivial solution; that is, we assumed that the system admitted $u=0$ as a solution. In this section we consider the saddle-node bifurcation for which $u=0$ is not an equilibrium solution of the governing equations. This type of bifurcation is characterised by the following system:

$$
d u / d t=C(\mu)+A(\mu)+F(\mu, u),
$$

where $F(\mu, 0)=\partial F(\mu, 0) / \partial u=0 ; A(\mu)$ and $C(\mu)$ are $n \times n(n \geq 2)$ matrices with the following properties

$$
\begin{aligned}
& C(0)=0, \\
& A(\mu) \zeta_{j}=\lambda_{j} \zeta_{j},(j=1,2, \ldots, n), \\
& \lambda_{1}(0)=0, \lambda_{1 \mu}(0)>0, \\
& \operatorname{Re} \lambda_{j}(0)<0,(j=2, \ldots, n) .
\end{aligned}
$$

$A(\mu), C(\mu)$ and $F(\mu, u)$ are assumed to be analytic functions of their respective arguments. Following the procedure of previous sections we allow $\mu$ to vary slowly with respect to $t$ and make a smooth transformation

$$
u=S v, \quad S=S(\tau), \quad \tau=\varepsilon t
$$

where both $S$ and $S^{-1}$ can be chosen to be smooth functions of $\tau$ such that

$$
\begin{gathered}
S^{-1} A S=B \\
B=\left(\begin{array}{cc}
\lambda_{1} & 0 \\
0 & B_{1}
\end{array}\right)
\end{gathered}
$$

and $B_{1}$ is an $(n-1) \times(n-1)$ matrix whose eigenvalues have negative real parts ( at $\tau=0$ ). In terms of the new variable $v$ the system (4.1) becomes

$$
d v / d t=C_{1}(\mu)-\varepsilon S^{-1} S_{\tau} v+B v+S^{-1} F(\mu, S v)
$$

where $C_{1}(\mu)=S^{-1} C(\mu)$. Again we make the decomposition

$$
v=a \zeta_{1}+z, \quad\left\langle z, \eta_{1}\right\rangle=0 .
$$

where

$$
\zeta_{1}=(1,0, \ldots, 0)^{\top}=\eta_{1}, \quad\left\langle\zeta_{1}, \eta_{1}\right\rangle=1
$$


are the eigenvectors of $B$ and $B^{*}$ (respectively) corresponding to the eigenvalue $\lambda_{1}$. With this decomposition (4.2) yields

$$
\begin{gathered}
d a / d t=b(\mu)+\lambda_{1} a-\varepsilon a \varphi_{1}-\varepsilon \beta z+F_{1}(\mu, a)+N_{1}(\mu, a, z), \\
d z / d t=B_{1} z+C_{2}(\mu)+\varepsilon a \varphi_{2}+\varepsilon B_{2} z+F_{2}(\mu, a)+N_{2}(\mu, a, z)
\end{gathered}
$$
where

$$
\begin{gathered}
b(\mu)=\left\langle C_{1}(\mu), \eta_{1}\right\rangle, \\
C_{2}(\mu)=C_{1}-b(\mu) \zeta_{1}, \\
\varphi_{1}(\mu)-\left\langle S^{-1} S_{\tau} \zeta_{1}, \eta_{1}\right\rangle, \\
\varphi_{2}=\varphi_{1}(\mu) \zeta_{1}-S^{-1} S_{\tau} \zeta_{1}, \\
\beta z=\left\langle S^{-1} S_{\tau} z, \eta_{1}\right\rangle, \\
B_{2} z=\beta z \zeta_{1}-S^{-1} S_{\tau} z, \\
F_{1}(\mu, a)+N_{1}(\mu, a, z)=\left\langle S^{-1} F(\mu, S v), \eta_{1}\right\rangle, \\
F_{2}(\mu, a)+N_{2}(\mu, a, z)=S^{-1} F(\mu, S v)-\left(F_{1}+N_{1}\right) \zeta_{1} .
\end{gathered}
$$

$F_{1}$ and $F_{2}$ are homogeneous in $a$ of degree $k \geq 2$ while $N_{1}$ and $N_{2}$ are nontrivial in $z$.

Proceeding as in previous sections we can make successive coordinate transformations of the form

$$
z^{\prime}=z+f_{1}(a, \tau), \quad a^{\prime}=a+\alpha a^{2}
$$

so that with a correct choice of $f_{1}$ and $\alpha,(4.3 \mathrm{a})$ and (4.3b) may be transformed to:

$$
\begin{aligned}
d a / d t & =b_{1}(\mu)-\varepsilon a \Psi_{1}+F_{3}(\mu, a)+N_{3}(\mu, a, z) \\
d z / d t & =\left(B_{1}+\varepsilon B_{2}\right) z+N_{4}(\mu, a, z)+O\left(\varepsilon^{n} a, a^{p}\right)
\end{aligned}
$$

where $n$ and $p$ can be chosen arbitrarily large. Here $b_{1}=O(\mu), N_{4}$ is related to $N_{2}$ of $(4.3 b), F_{3}=O\left(|a|^{2}\right)$ and $N_{3}$ is nontrivial in $z$. Since the eigenvalues of the matrix $B_{1}+\varepsilon B_{2}$ have negative real parts (in a small neighbourhood about $\tau=0$ ) we can, once again, obtain an estimate on the magnitude of $z$ :

$$
z=O\left(\varepsilon^{n}|a|,|a|^{p}\right) .
$$

Thus (4.4a) may be rewritten as:

$$
d a / d t=b_{1}+\alpha a^{2}+O\left(\varepsilon a, a^{3}\right)
$$

where $\alpha$ is an $O(1)$ quantity. Here we have used the fact that $\mu=\mu(\tau)$. For simplicity we assume the canonical case $b_{1}=b \tau, b>0$ and $\alpha<0$. In this case (4.5) has leading order outer solutions

$$
a^{2} \sim-b \tau / \alpha
$$

which exist only for $\tau>0$ (since $\alpha b<0$ ). 
Restricting our attention to a neighbourhood of the origin we rescale

$$
a=\varepsilon^{1 / 3} X /\left(b|\alpha|^{2}\right), \quad \tau=\varepsilon^{2 / 3} T /(b|\alpha|) .
$$

To leading order in $\varepsilon,(4.5)$ then yields

$$
d X / d T=T-X^{2} .
$$

Equation (4.7) arises in the study of jump phenomena in ordinary differential equations when the parameter governing the jump is varied slowly through its critical value (at which the jump occurs). Haberman [4] obtains (4.7) as the equation governing the inner solution of an inner/outer asymptotic expansion for jump phenomena governed by first order nonlinear differential equations. Kapila [7] derives (4.7) as the equation governing the transition (jump) between two "steady-state" solutions of a model Arrhenius system.

We note that the transformation $X=w^{-1} d w / d T$ reduces (4.7) to the Airy differential equation $d^{2} w / d T^{2}=T w$ whose general solution may be written as $w=c_{1} A i(T)+c_{2} B i(T)$ where $A i(T), B i(T)$ are the Airy functions (see Abramowitz and Stegun [1]) and $c_{k}$ are arbitrary constants. Thus the general solution to (4.7) can be written as

$$
X=\frac{A i^{\prime}(T)+C B i^{\prime}(T)}{A i(T)+C B i(T)}
$$

where $C=c_{2} / c_{1}$.

The solution $X$ has an infinite number of singularities (simple poles) at values $T_{k}, T_{k+1}<T_{k}(k=0,1,2, \cdots)$ satisfying

$$
A i\left(T_{k}\right)+C B i\left(T_{k}\right)=0 .
$$

We can classify the solutions (4.8) according to the value of the constant $C$. (a) $C=0$. Then $T_{0}=\alpha_{1}$ where $\alpha_{1}=-2.33810741$ is the first zero of the function $\operatorname{Ai}(T)$. In this case we have (see [1])

$$
X \sim-\sqrt{T} \text { as } T \rightarrow+\infty .
$$

This corresponds to a transition from the fully nonlinear outer solution to the unstable branch of the outer solution as $T \rightarrow \infty$.

(b) $C>0$. Then the first singularity occurs at $T_{0}, \alpha_{1}<T_{0}<\beta_{1}<0$, where $\beta_{1}=-1.17371322$ is the first zero of the function $B i(T)$. In this case

$$
X \sim \sqrt{T} \text { as } T \rightarrow+\infty
$$

This corresponds to a transition from the fully nonlinear outer solution to the stable branch of the outer solution as $T \rightarrow \infty$. 


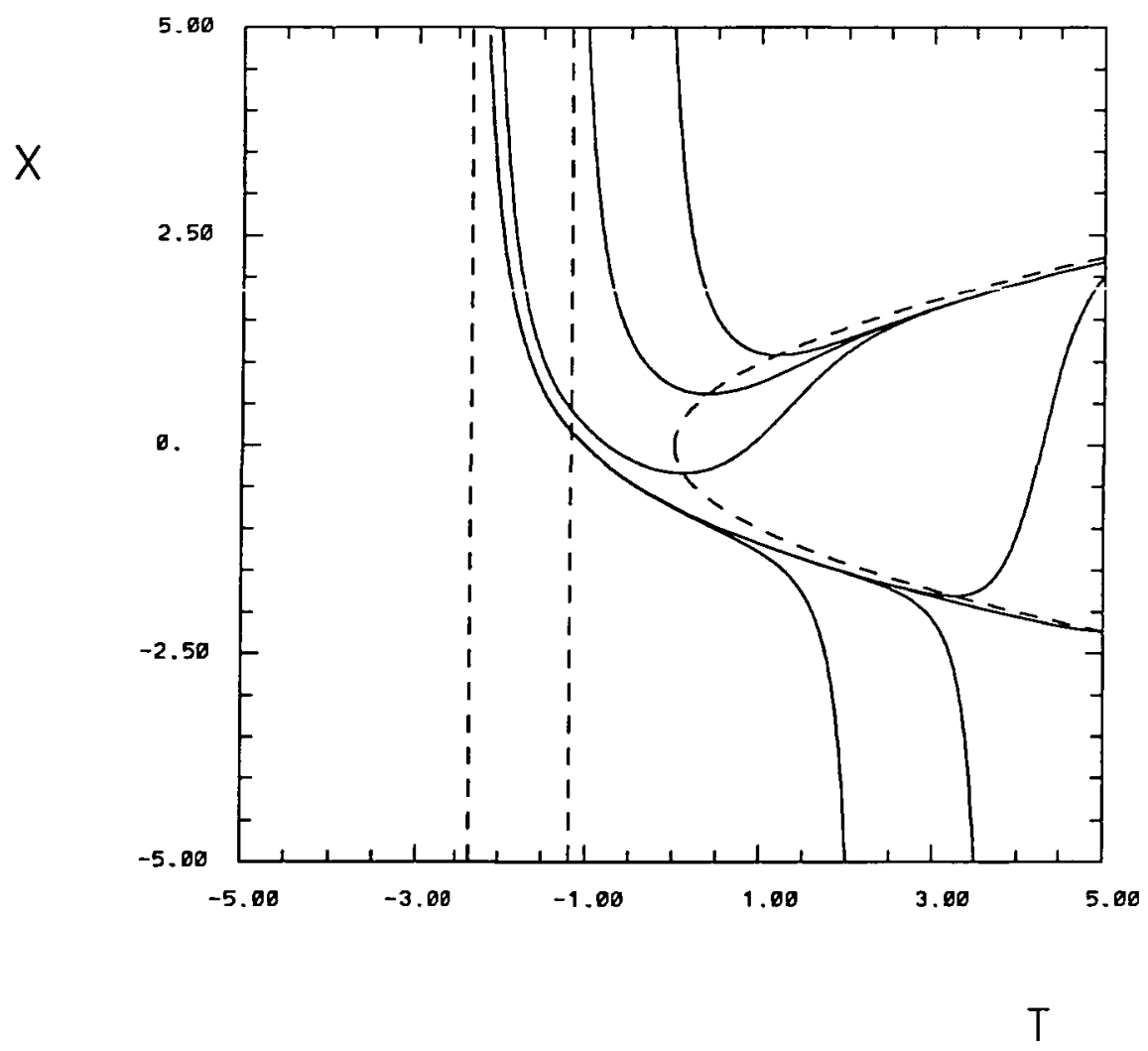

Figure 5. Numerical solutions of (4.7) (solid lines). Dashed curve $X= \pm T^{1 / 2}$. First zeroes $\alpha_{1}$ and $\beta_{1}$ of $A i(T)$ and $B i(T)$, respectively, are marked as vertical dashed lines.

(c) $C<-A i(0) / B i(0)<0$. In this case $\alpha_{1}<\beta_{1}<T_{0}<0$ and again

$$
X \sim \sqrt{T} \text { as } T \rightarrow+\infty,
$$

a transition from the fully nonlinear outer solution to the stable branch of the outer solution as $T \rightarrow \infty$.

(d) $0>C>-A i(0) / B i(0)$. Now $T_{0}>0$ and the solution (4.8) connects the singularity at $T_{1}<0$ with the singularity at $T_{0}>0$ (see Figure 5).

In Figure 5 we present numerical solutions of (4.7) exhibiting the general behaviour of the solutions (4.8) of (4.7).

The explosion in the solutions at those times $T_{k}$ given by (4.9) are related to the jump phenomena considered by Haberman [4]. It can be shown that these singularities connect the inner solution governed by (4.7) to the fully nonlinear outer problem. This is achieved by rescaling the time about the 
point at which the singularity occurs. The appropriate scaling is

$$
t=\varepsilon^{-1 / 3} T_{k}+\tau \text {. }
$$

The new outer solution is then governed (to leading order) by a fully nonlinear problem (for details see Haberman [4]). The other singularities which occur at negative times are, in fact, artifacts of the inner/outer asymptotic expansion and may be removed by matching the inner solution to the appropriate outer solution.

\section{Discussion}

We have shown that in most cases considered there is a smooth transition to the bifurcated solutions when the parameter is varied slowly. The higher dimensional problems have been reduced to problems similar to those which have been studied by Haberman [4] and other authors.

The case of Hopf bifurcation is new. However, it has been shown that this case closely parallels that of the pitchfork bifurcation problem for first order ordinary partial differential equations as studied by Haberman.

It should be noted that at those points at which the singularities occur our original scalings are no longer valid. At these points one must again rescale. However, since this analysis has been carried out by Haberman, we will not reproduce it here except to mention that these singularities correspond to a transition to a higher branch of the bifurcated solution.

\section{Acknowledgements}

The authors would like to thank Professor R. Haberman for many special discussions and comments concerning this work. During the course of this work J. Denier was the holder of a Commonwealth Postgraduate Research Award.

\section{References}

[1] M. Abramowitz and I. A. Stegun, Handbook of mathematical functions (Washington: National Bureau of Standards, 1965).

[2] T. Erneux and P. Mandel, "Imperfect bifurcation with a slowly-varying control parameter", SIAM J. Appl. Math. 46 (1986) 1-15.

[3] J. Guckenheimer and P. Holmes, Nonlinear oscillations, dynamical systems, and bifurcations of vector fields (Springer-Verlag, New York, 1983). 
[4] R. Hauberman, "Slowly varying jump and transition phenomena associated with algebraic bifurcation problems", SIAM J. Appl. Maths. 37 (1979) 69-106.

[5] P. Hall, "On the nonlinear stability of slowly varying time-dependent viscous flows", $J$. Fluid Mech. 126 (1983) 357-368.

[6] G. Iooss and D. D. Joseph, Elementary stability and bifurcation theory (Springer-Verlag, New York, 1980).

[7] A. K. Kapila, “Arrhenius systems: dynamics of jump due to the slow passage through criticality", SIAM J. App!. Math. 41 (!981) 29-42.

[8] N. R. Lebovitz and R. J. Schaar, "Exchange of stabilities in autonomous systems", Stud. Appl. Maths. 54 (1975) 229-260.

[9] N. R. Lebovitz and R. J. Schaar, "Exchange of stabilities in autonomous systems-II. Vertical bifurcation ", Stud. Appl. Maths. 56 (1977) 1-50.

[10] L. A. Rubenfeld, "A Model bifurcation problem exhibiting the effects of slow passage through critical", SIAM J. Appl. Math. 37 (1979) 302-306.

[11] S. Schecter, "Persistent unstable equilibria and closed orbits of a singularly perturbed equation", J. Diff. Equations 60 (1985) 131-141. 